\title{
Longitudinal fields of quantized Laguerre-Gaussian modes
}

\author{
Kayn A. Forbes, Dale Green, Garth A. Jones \\ School of Chemistry, University of East Anglia, Norwich NR4 7TJ, United Kingdom \\ k.forbes@uea.ac.uk
}

\begin{abstract}
Longitudinal fields of quantized Laguerre-Gaussian modes are derived and studied with respect to the paraxial parameter $k w_{0}$. Generally these longitudinal fields are ignored in the paraxial approximation, however it shown how they can be become important even for relatively large values of $k w_{0}$. This is in contrast to unstructured laser light, e.g. a Gaussian beam, where the magnitude of longitudinal components only become important under strong-focusing of the source. The unique effects stem specifically from the optical angular momentum, both orbital and spin, of optical vortex light, and include spin-orbit interactions in freely-propagating circularly-polarized vortices in free-space. The contribution that longitudinal fields make to the rate of single-photon absorption is calculated, highlighting that for optical vortices they cannot be neglected in general.
\end{abstract}

Keywords: Non-paraxial optics, twisted light, optical vortex, spin-orbit interactions of light, optical orbital angular momentum, light-matter interactions

\section{INTRODUCTION}

Exact plane-wave solutions to the Maxwell and Helmholtz equations are most commonly utilized to provide a theoretical understanding of light-matter interactions ${ }^{1}$. Beyond these completely transverse plane-wave solutions, the Helmholtz wave equation also permits 'laser beam' solutions, e.g. Gaussian modes, however, these and the ensuing solutions to the Maxwell equations are only approximate. A key property of such solutions is that they are not strictly transverse in free space, and the electric and magnetic fields generally must have non-zero components parallel to the direction of propagation to ensure $\nabla \cdot E=0$ and $\nabla \cdot B=0^{2}$ : viz longitudinal fields.

Lax $e t a l^{3}$ highlighted how the paraxial solutions to the scalar wave equation consist of a purely transverse zeroth-order field (we denote $T_{0}$ ) and smaller, first-order, longitudinal components $\left(L_{1}\right)$ whose magnitude for Gaussian-type beams depends on the paraxial factor $\left(k w_{0}\right)^{-1}$ where $k=2 \pi / \lambda$ is the wave number and $w_{0}$ the beam waist at the focal point. Therefore, it is understood that the importance of longitudinal fields for general laser modes are correlated to the degree of focusing, where strongly-focused beams exhibit larger longitudinal components in their electromagnetic fields.

A realization that has led to a highly active area of modern optics is that longitudinal components to the total fields are not simply just quantitative corrections to $\mathrm{T}_{0}$ fields but can exhibit highly distinct properties that influence propagation characteristics of the light or the ensuing interactions with matter. Focused circularly-polarized Gaussian beams for example can induce orbital motion of trapped particles due to the spin-to-orbital angular momentum conversion ${ }^{4,5}$.

Twisted light beams or optical vortices are an extremely well-studied type of structured laser light due to their rich angular momentum properties. For paraxial vortex modes, the individual photons can exhibit a spin angular momentum (SAM) $\sigma \hbar$, where $\sigma= \pm 1$, as well as additional units of orbital angular momentum (OAM) $\ell \hbar$, where $\ell \in \mathbb{Z}$, per photon. Most 
studies have been concerned with the angular momentum properties of non-paraxial and longitudinal fields of twisted light, such as spin-orbit interactions of light (SOI) and the transfer to particles to cause mechanical motion ${ }^{6-8}$. The application of twisted light in spectroscopic applications is a burgeoning area of research ${ }^{9}$. The potentially unique role that longitudinal fields can play in these applications has previously been highlighted for highly-focused Laguerre-Gaussian (LG) beams ${ }^{10,11}$, and the transfer of optical OAM to an atom can only be correctly accounted for quantitatively if longitudinal fields of the input optical vortex are accounted for ${ }^{12}$.

Here we derive the quantum electromagnetic field mode operators for LG beams that include $\mathrm{L}_{1}$ components in addition to the $\mathrm{T}_{0}$ fields. We highlight numerically that there are two distinct factors that influence the magnitude of longitudinal fields of optical vortices; firstly the well-known fact that a larger degree of focusing increases the longitudinal fields through a paraxial parameter $\left(k w_{0}\right)^{-1}$ weighting factor; but secondly that the angular momenta of optical vortices produces longitudinal fields that cannot be neglected for paraxial beams, highlighting both the quantitative and qualitative necessity of their inclusion.

\section{LONGITUDINAL COMPONENT OF A QUANTIZED LG MODE}

In the Power-Zienau-Woolley (PZW) formulation of quantum electrodynamics (QED) ${ }^{13,14}$ the electromagnetic field operators that couple to matter are the electric displacement field $\boldsymbol{d}^{\perp}(\boldsymbol{r})$ and magnetic field $\boldsymbol{b}(\boldsymbol{r})$. The superscript $\perp$ on $\boldsymbol{d}^{\perp}(\boldsymbol{r})$ is with reference to the fact that for a neutral system $\nabla \cdot \boldsymbol{d}=0^{15}$, and highlights that in the PZW formulation of QED all coulombic interactions are mediated by transverse photons ${ }^{16}$.

The most utilized of optical vortices are the Laguerre-Gaussian modes, which are solutions to the paraxial equation in cylindrical coordinates. As such, longitudinal components are of LG modes are generally neglected under the guise of the paraxial approximation. The electric displacement field mode expansion operator for LG modes in the long Rayleigh range limit $z_{R} \gg z$ has previously been derived $^{17}$, and is given as

$\boldsymbol{d}_{x, y}^{\perp}(\boldsymbol{r})=\sum_{k, \sigma, \ell, p} \frac{\tilde{\Omega}}{\sqrt{2}}\left[(\hat{\boldsymbol{x}}+i \sigma \hat{\boldsymbol{y}}) a_{|\ell|, p}{ }^{(\sigma)}(k \hat{z}) f_{|\ell|, p}(r) \mathrm{e}^{i(k z+\ell \phi)}-H . c.\right]$,

where $k$ is the wave number; $(\hat{\boldsymbol{x}}+i \sigma \hat{\boldsymbol{y}})$ is the polarization vector where $\sigma= \pm 1$ for left- and right-handed circularlypolarized light, respectively; $a^{(\sigma)}(\boldsymbol{k})$ is the annihilation operator; $\tilde{\Omega}$ is the normalization constant $\tilde{\Omega}=i\left(\hbar c k \varepsilon_{0} / 2 A_{\ell, p}^{2} V\right)^{1 / 2}$ with $V$ the quantization volume; $f_{|\ell|, p}(r)=\frac{C_{p}^{|\ell|}}{w_{0}}\left(\frac{\sqrt{2} r}{w_{0}}\right)^{|\ell|} \mathrm{e}^{-\frac{r^{2}}{w_{0}^{2}}} L_{p}^{|\ell|}\left[\frac{2 r^{2}}{w_{0}^{2}}\right]$ is a radial distribution function where $C_{p}^{|\ell|}$ is a constant and $L_{p}^{|\ell|}$ is the associated Laguerre polynomial, and H.c. stands for the Hermitian conjugate. The subscript $x, y$ on $\boldsymbol{d}_{x, y}^{\perp}(\boldsymbol{r})$ refers to the fact the fields are purely transverse to the Poynting vector, which for plane-waves in free space exactly conincides with the wave vector $\boldsymbol{k}$. 
In the plane-wave solutions commonly used, the total field is simply $\boldsymbol{d}^{\perp}(\boldsymbol{r})=\boldsymbol{d}_{x, y}^{\perp}(\boldsymbol{r})$. However, for any beam-like solution to the Helmholtz equation, the zeroth-order $\mathrm{T}_{0}(1)$ is only an approximation, and the total field is $\boldsymbol{d}^{\perp}(\boldsymbol{r})=\boldsymbol{d}_{x, y}^{\perp}(\boldsymbol{r})+\hat{z} d_{z}^{\perp}(r)$ . The most direct method to calculate the $\mathrm{L}_{1}$ components is using the transversality conditions of Maxwell's equations ${ }^{8,12}$. In order to generate the $\mathrm{L}_{1}$ terms for $\boldsymbol{d}^{\perp}(\boldsymbol{r})$ we use Gauss's Law: $\boldsymbol{\nabla} \cdot \boldsymbol{d}=0$. Thus the $z$ components $d_{z}^{\perp}(r)$ of the field can be determined via

$$
\int \nabla^{\perp} \cdot \boldsymbol{d}_{x, y}^{\perp}(\boldsymbol{r}) \mathrm{d} z=\sum_{k, \sigma, \ell, p} \frac{\tilde{\Omega}}{\sqrt{2}}\left[\frac{i}{k}\left(\frac{\partial}{\partial x}+i \sigma \frac{\partial}{\partial y}\right) f_{|\ell|, p}(r) a_{|\ell|, p}{ }^{(\sigma)}(k \hat{z}) \mathrm{e}^{i(k z+\ell \phi)}-H . c .\right] .
$$

Using Cartesian to cylindrical coordinate transformations produces the following mode expansion which now includes the additional first-order longitudinal corrections to the zeroth-order transverse field ${ }^{18}$ :

$$
\boldsymbol{d}^{\perp}(\boldsymbol{r})=\sum_{k, \sigma, \ell, p} \frac{\tilde{\Omega}}{\sqrt{2}}\left[\left\{(\hat{\boldsymbol{x}}+i \sigma \hat{\boldsymbol{y}})+\frac{i}{k}\left(\frac{\partial}{\partial r}-\ell \sigma \frac{1}{r}\right) \mathrm{e}^{i \sigma \phi} \hat{z}\right\} f_{|\ell|, p}(r) a_{|\ell|, p}{ }^{(\sigma)}(k \hat{z}) \mathrm{e}^{i(k z+\ell \phi)}-H . c\right] .
$$

There are some interesting features of (3): For $\ell=0$, one of the longitudinal terms is zero, but the other survives, highlighting how even longitudinal fields of a circularly-polarized Gaussian beam (i.e LG $_{0}^{0}$ ) exhibit a vortex of charge one structure in the $z$ direction through the phase factor $\mathrm{e}^{i \sigma \phi}$. This SOI is known to occur in freely-propagating, nonparaxial beams of light ${ }^{19}$. Another SOI is evident when the incident beam does have an LG structure and accompnaying OAM. For $\ell=\sigma$ the longitudinal fields form a vortex of charge 2; whereas for $\ell=-\sigma$ the beam exhibits a Gaussian structure with maximum intensity along the beam axis. This form of parallel and anti-parallel SAM and OAM projections, respectively, has been utilized in numerous studies ${ }^{20,21}$.

For the vast majority of applications, the electric field is sufficient to describe light-matter interactions. However, magnetic interactions can become important in the correct settings, such as in chiral optics ${ }^{22}$. The magnetic field mode expansion is found using a similar approach, but with the aid of $\boldsymbol{\nabla} \cdot \boldsymbol{b}=0$ :

$$
\boldsymbol{b}(\boldsymbol{r})=\sum_{k, \sigma, \ell, p} \frac{\tilde{\Omega}}{\sqrt{2} c}\left[\left\{(\hat{\boldsymbol{y}}-i \sigma \hat{\boldsymbol{x}})+\frac{1}{k}\left(\sigma \frac{\partial}{\partial r}-\frac{\ell}{r}\right) \mathrm{e}^{i \sigma \phi} \hat{z}\right\} f_{|\ell|, p}(r) a_{|\ell|, p}{ }^{(\sigma)}(k \hat{z}) \mathrm{e}^{i(k z+\ell \phi)}-H . c\right] .
$$

The first-order longitudinal components of the magnetic field (4) are wholly dependent on the angular momentum properties of the light, e.g. according to (4) a linearly-polarized Gaussian beam $(\sigma, \ell, p=0)$ possesses no longitudinal magnetic fields, only for strongly-focused light where higher-order terms (second-order and above) contribute do longitudinal magnetic fields arise in this case. 


\section{INFLUENCE OF PARAXIAL FACTOR}

The validity of the paraxial approximation has been questioned by numerous authors previously ${ }^{23-25}$, and even with respect to optical vortices interacting with atoms ${ }^{12,26,27}$. These works predominantly concentrated on specific systems. We now highlight how the reliance on thetransverse (zeroth-order) is problematic in general for paraxial optical vortices. To aid clarity of the analysis as well as to readily yield analytic results, we focus on LG modes where $p=0$. Furthermore we note that these are the most utilized modes in experiments.

The electric displacement field (3) takes on the following form when $p=0$ and the differentiation with respect to $r$ is carried out (remembering we are working within the long Rayleigh range limit; we have also dropped the obvious dependencies for notational clarity):

$\boldsymbol{d}^{\perp}(\boldsymbol{r})=\sum_{k, \sigma, \ell} \frac{\tilde{\Omega}}{\sqrt{2}}\left[\left\{(\hat{\boldsymbol{x}}+i \sigma \hat{\boldsymbol{y}})+\frac{i}{k}\left(\frac{|\ell|}{r}-\frac{\ell \sigma}{r}-\frac{2 r}{w_{0}^{2}}\right) \mathrm{e}^{i \sigma \phi} \hat{z}\right\} f_{|\ell|, 0} a \mathrm{e}^{i(k z+\ell \phi)}-H . c\right]$.

The magnitude of the last longitudinal term is weighted by the factor $2 r / k w_{0}^{2}$, similarly to that of a Gaussian beam, where $k w_{0}^{2}$ is sometimes referred to as the diffraction length ${ }^{3}$. This generally small contribution (for weakly-focused beams ${ }^{28}$ ) to the longitudinals fields is independent of the angular momentum properties of the field, and is exhibited by any field mode which possesses a Gaussian factor.

Interestingly, there are an additional two terms that depend on the angular momentum properties of the beam. First there is the contribution dependent on the factor $|\ell| / k r$ which is the absolute value of the skew angle of the Poynting vector at a given location ${ }^{29}$. If the light also posesses SAM, there is the additional skew-angle-like term dependent on $\ell \sigma / k r$, which thus includes the mixing of helicity and topological charge values. The skew angle term dependent on $|\ell|$ reveals that a longitudinal field exists for a non-circularly polarized $(\sigma=0)$ LG beam, whilst the second term signififes a SOI of light in freely-propagating circularly polarized optical vortices in free-space, a phenomenon highlighted some time ago ${ }^{30}$ but has seemingly received relatively little attention.

The notion that longitudinal fields for optical vortices may be neglected unless the light is non-paraxial pervades the literature, e.g. ${ }^{8,31-33}$, and is a commonly held misconception, likely stemming from the fact that this is generally true for paraxial laser modes that do not possess OAM. The intensity distributions as a function of beam waist of a variety of LG modes where the first-order longitudinal fields have been included with the generally used zeroth-order tranvserse fields is given in Figure 1 and Figure 2. 


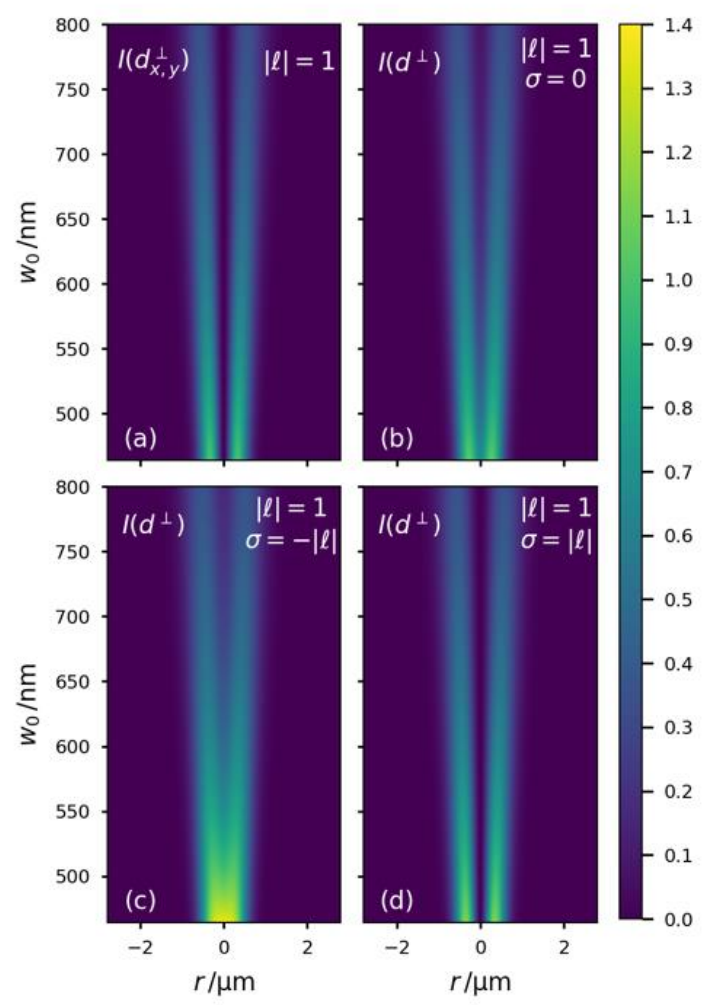

Figure 1: Intensity distributions for an LG beam with $|\ell|=1$ for a changing beam waist a) the intensity contains only the transverse field components b) the total intensity including longitudinal contributions for $\sigma=0$ c) Total intensity for antiparallel OAM and SAM and d) Total intensity for parallel OAM and SAM. The range of beam waists used in Figure 1 and 2 correspond to $4 \leq k w_{0} \leq 20$.

The range of beam waists used in Figure 1 and 2 correspond to $4 \leq k w_{0} \leq 20$, i.e. within the range that a paraxial solution to the wave equation yields a correct descritpion of the light ${ }^{24}$. Just as it is well-established that longitudinal fields cannot be neglected when unstructured (non-OAM) light is very strong- focused because terms dependent on factors like $2 r / k w_{0}^{2}$ become important, Figure 1 shows that certain contributions to longitudinal fields for paraxial optical vortices cannot likewise be neglected.

The importance of these fields are still bound to specific scenarios due to the dependence on the radial distribution function, beam waist, optical angular momentum, and in comparison to the zeroth-order transverse fields are weighted by the wavelength (inverse wave number).

Whilst it is true that these terms do indeed become larger the more focused the beam is, Figure $1 \mathrm{~b}$ and $1 \mathrm{c}$ clearly highlights how they manifest even in LG modes that are not extremely focused, particularly in the so-called 'vortex core' which evidently is not truly empty for a range of parameters. Furthermore, if the twisted light is also circularly-polarized the effects become more significant than if $\sigma=0$, and in the case of anti-parallel SAM and OAM the paraxial approximation significantly fails for the whole range of values of $k w_{0}$ (Figure 1c). As Figure 2 shows, for higher values of $|\ell|$ the on-axis intensity distribution vanishes for paraxial beams, and the total field intensity distribution much more resembles the transverse only components, but the transverse only description still differs from the total field, especially for the lower 
values of $k w_{0}$ when $\ell= \pm 2, \sigma=\mp 1$ as in Figure 2c. Although we have shown that the inclusion of solely zeroth-order transverse fields is not in general adequate for paraxial LG modes, it is clear that the paraxial approximation most significantly fails quantitatively for $\ell= \pm 1, \sigma=0$ and $\ell= \pm 1, \sigma=\mp 1$.

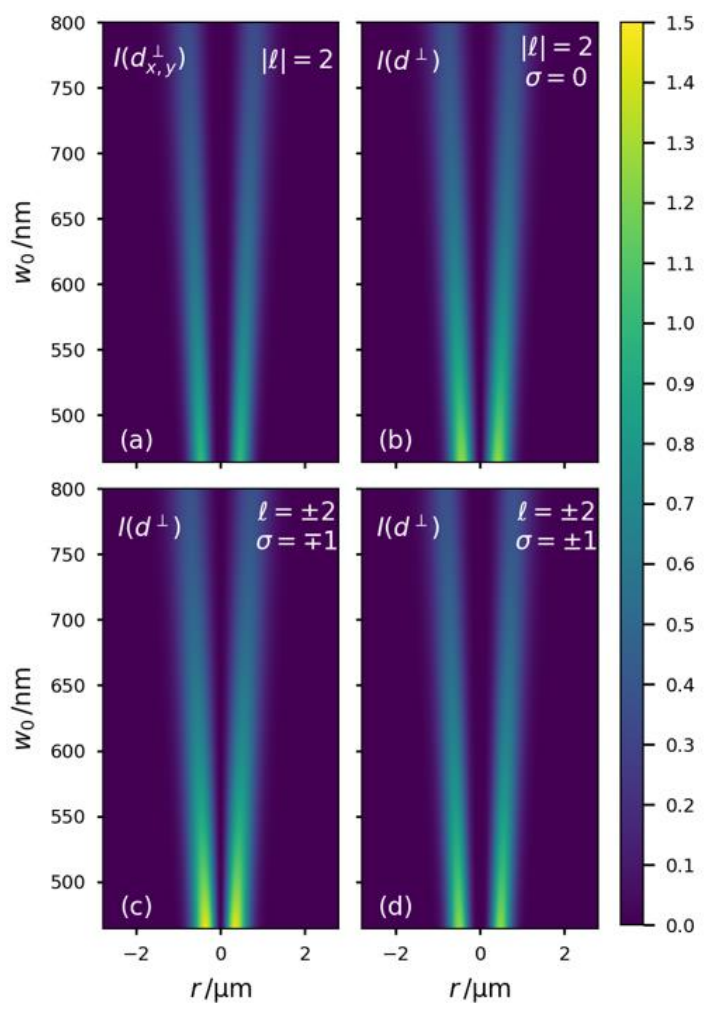

Figure 2: Intensity distributions for an LG beam with $|\ell|=2$ for a changing beam waist (wavelength $729 \mathrm{~nm}$ ) a)-d) correspond to the same conditions as those in Figure 1, the range of $k w_{0}$ is also the same.

\section{APPLICATION TO ABSORPTION}

Single-photon absorption is the simplest optical process, and is therefore an appropriate initial case to investigate the role of longitudinal fields in light-matter interactions. We may calculate the matrix element (or quantum amplitude) $M_{F I}$ of single-photon absorption in the electric dipole approximation using standard time-dependent perturbation methods, with $M_{F I}$ given by: $\left\langle F\left|H_{\mathrm{int}}\right| I\right\rangle=\left\langle F\left|-\varepsilon_{0}^{-1} \boldsymbol{\mu} \cdot \boldsymbol{d}^{\perp}(\boldsymbol{r})\right| I\right\rangle$, where the initial state vector $|I\rangle=\left|n(k, \sigma, \ell, p) ; E_{0}\right\rangle$ consists of $n$ photons in the $\mathrm{LG}$ mode $(k, \sigma, \ell, p)$ and the material in the ground state $E_{0}$; the final state is given by $|F\rangle=\left|(n-1)(k, \sigma, \ell, p) ; E_{\alpha}\right\rangle$, where the mode has lost a photon and the material now exists in the excited state denoted by $\alpha ; \boldsymbol{\mu}$ is the electric-dipole transition moment operator. 
Using the mode expansion for the electric displacement field operator (3), the matrix element is

$M_{F I}=-\tilde{\Omega} \sqrt{n}\left[e_{i}^{\mathrm{L} / \mathrm{R}}+\frac{i}{\sqrt{2} k}\left(\frac{\partial}{\partial r}-\frac{\ell \sigma}{r}\right)\right] \hat{z}_{i} \mathrm{e}^{i \sigma \phi} f \mu_{i}^{\alpha 0} \mathrm{e}^{i(k z+\ell \phi)}$,

where we now use suffix notation for tensor quantities and $e_{i}^{\mathrm{L} / \mathrm{R}}=2^{-1 / 2}(\hat{x}+i \sigma \hat{y})_{i}$ is the polarization vector for circularly polarized light. As is standard ${ }^{16}$, the matrix element is inserted into the Fermi rule to yield the rate $\Gamma$ of single-photon absorption as:

$\Gamma \propto\left|M_{F I}\right|^{2}=n \tilde{\Omega}^{2}\left[e_{i}^{\mathrm{L} / \mathrm{R}} \bar{e}_{j}^{\mathrm{L} / \mathrm{R}} f^{2}+\frac{1}{k r}\left(\sigma r f f^{\prime}-\ell f^{2}\right) \hat{z}_{i}\left(\hat{y}_{j} \cos \phi-\hat{x}_{j} \sin \phi\right)+\frac{\hat{z}_{i} \hat{z}_{j}}{2 k^{2} r^{2}}\left(-2 \ell \sigma r f f^{\prime}+\left(r f^{\prime}\right)^{2}+(\ell f)^{2}\right)\right] \mu_{i}^{\alpha 0} \bar{\mu}_{j}^{\alpha 0}$.

The prime superscripts in (7) denote partial differentiation with respect to $r$. The first term in square brackets in (7) is the standard rate of absorption via the $\mathrm{T}_{0}$ electric field, and it is therefore evident through the multitude of additional terms that accounting for longitudinal fields offer numerous additional optical interactions and qualitative corrections to the zeroth-order fields. The terms dependent on $\phi$ stem from the interferences between the transverse and longitudinal fields, and if a full beam-profile integration over $\phi$ is carried out (i.e. $\phi_{0} \rightarrow \phi_{0}+2 \pi$ ) these effects vanish. To render these observable the signals stemming from individual nanostructures or sub-domains must be resolved. The final terms are all purely longitudinal in nature, and their importance is determined by the factors discussed in the previous section.

The rate of absorption given by (7) specifically corresponds to a nanostructure with a fixed orientation with respect to the input optical axis. It is clear that that the $\mathrm{T}_{0}$ components can excite electric dipole transitions which must have allowed components in $x, y$-directions, whereas excitation through the pure longitudinal terms excite transitions which must exhibit components along the direction of propagation $z$. A similar scheme was used to map the fluorescence of molecules with specific orientations in order to precisely determine of the structure of electromagnetic fields for vector vortex beams ${ }^{34}$. It is also worthwhile noting that individual contributions to the total rate depend on $\sigma$, i.e. the handedness of the input circular polarization; the sign of $\ell$, i.e. the handedness of the optical vortex; and the product of the two $\ell \sigma$. Generally materials, such as molecular matter, need to be chiral in order to exhibit differential effects with respect to the sign of $\sigma$ (optical activity) through higher-order magnetic dipole and electric quadrupole interferences with the electric dipole transitions, and are thus usually weak effects ${ }^{35}$. The rate (7) tells us that in principle it is possible that photon absorption through purely electric-dipole transitions can yield a small differential rate for optical vortices with different helical wavefront and polarization handedness. These differential rates that depend on the optical handedness here are comparable to standard optical activity of circularly polarized light, but in comparison to these traditional chiroptical interactions that probe the local helicity of light, the phenomena here are clearly spatial effects related to a radially varying and optical angular momentum-dependent intensity structure ${ }^{36}$.

Orientational averaging is done using standard methods ${ }^{37}$, namely for a second rank tensor $\left\langle\mu_{i} \bar{\mu}_{j}\right\rangle=\frac{1}{3} \delta_{i j} \mu^{\alpha 0} \cdot \bar{\mu}^{\alpha 0}$ :

$\langle\Gamma\rangle \propto \frac{n \tilde{\Omega}^{2}}{3}\left(f^{2}-\frac{\ell \sigma}{k^{2} r} f f^{\prime}+\frac{1}{2 k^{2}} f^{\prime 2}+\frac{\ell^{2}}{2 k^{2} r^{2}} f^{2}\right)\left|\mu^{\alpha 0}\right|^{2}$. 
The total averaged rate (8) and its individual components are plotted in Figures 3-5 for different combinations of angular momentum. It is interesting to note how an orientational average of the individual material particles also leads to the interference terms averaging to zero. Clearly the different material transition moment orientational dependences of (7) to the transverse and longitudinal fields are also lost. The SOI term dependent on $\ell \sigma$ still survives, so that even randomly oriented molecules will still exhibit a small difference in the rate of absorption depending on the polarization and wavefront handedness - this is most clear by comparing Figure $4 \mathrm{e}$ and $5 \mathrm{c}$ for a given position $r$ (though note how the light must possess both SAM and OAM in this case).

It is clear to see that the rate of absorption has an acute dependence on radial position, but also the total rate is altered by the longitudinal fields even for paraxial vortices. The results displayed in Figure 3-5 corresponds to a laser beam propagating at the limit of the paraxial validity $k w_{0} \approx 4$. Note that Quinteiro et al. ${ }^{12}$ highlighted how longitudinal fields in the specific case of parallel and anti-parallel SAM and OAM had a significant influence on atomic electric quadrupole transitions even when $k w_{0} \approx 23$ (see our Figure 1c).
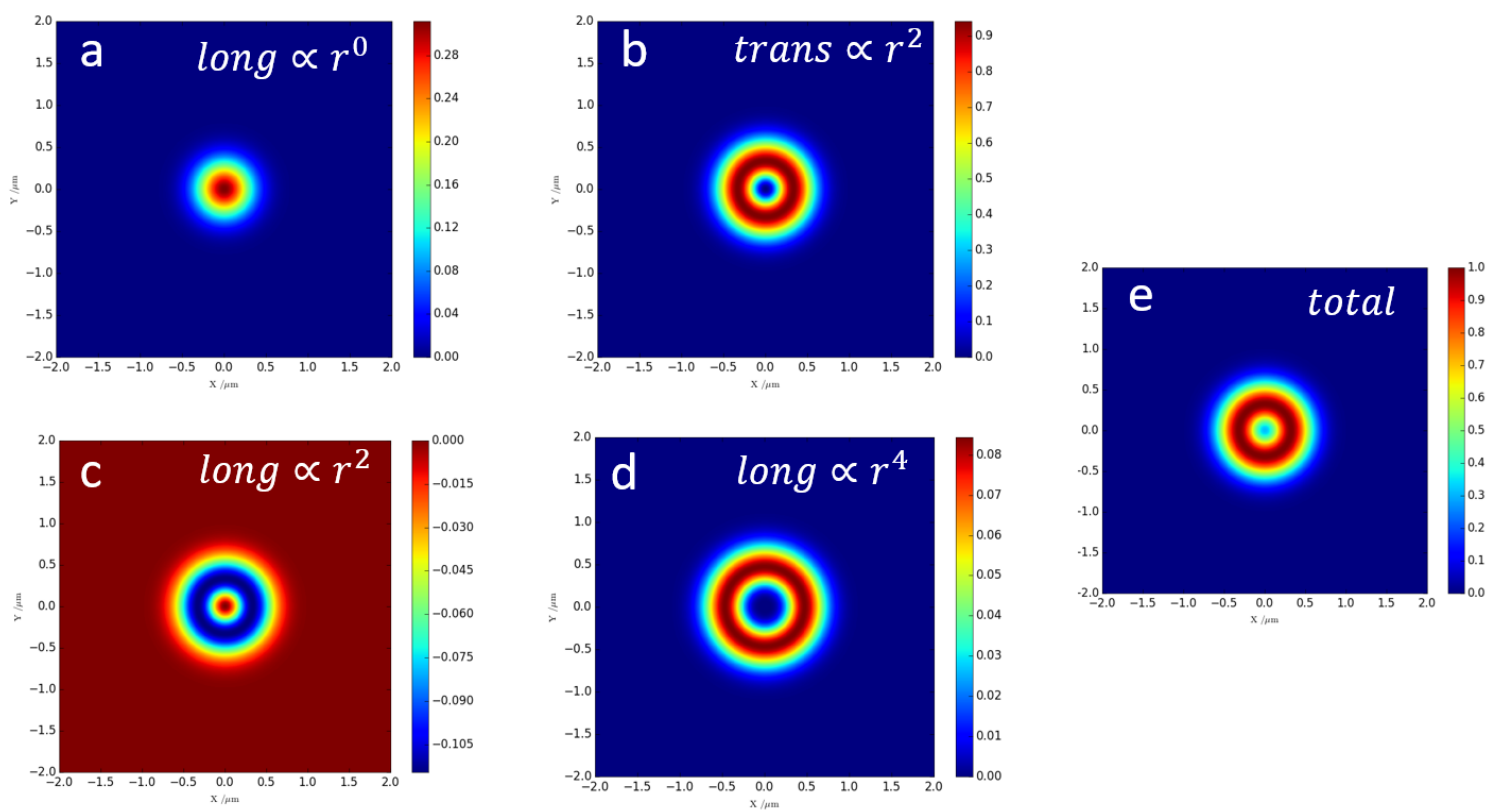

Figure 3: Normalised plots of the individual and total contributions to the rate of single-photon absorption (Eq.(8)) for $|\ell|=1, \sigma=0$. ( $p=0$, $\left.k w_{0} \approx 4\right)$. 

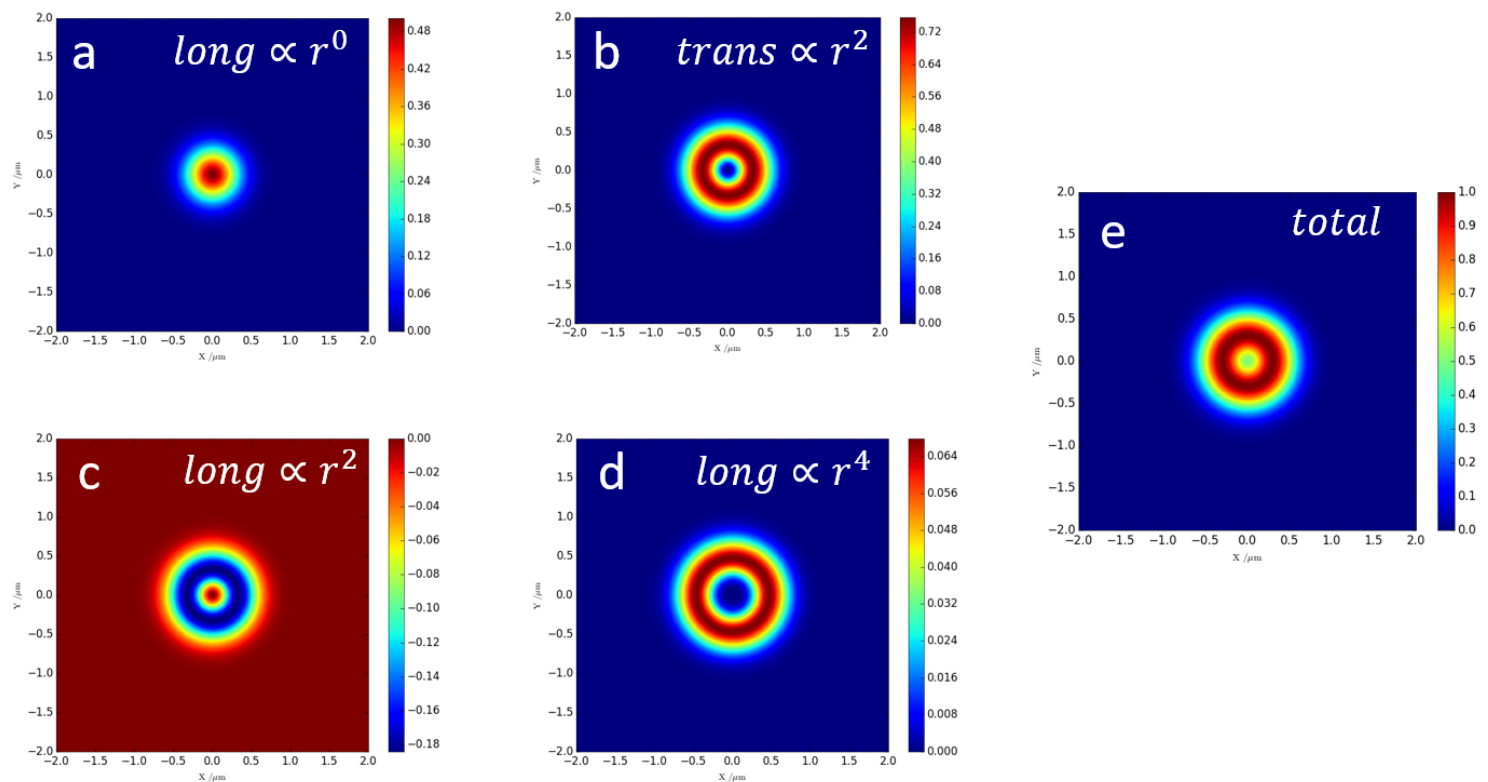

Figure 4: Normalised plots of the individual and total contributions to the rate of single-photon absorption (Eq.(8)) for $\ell= \pm 1, \sigma=\mp 1$. ( $p=0$, $\left.k w_{0} \approx 4\right)$.
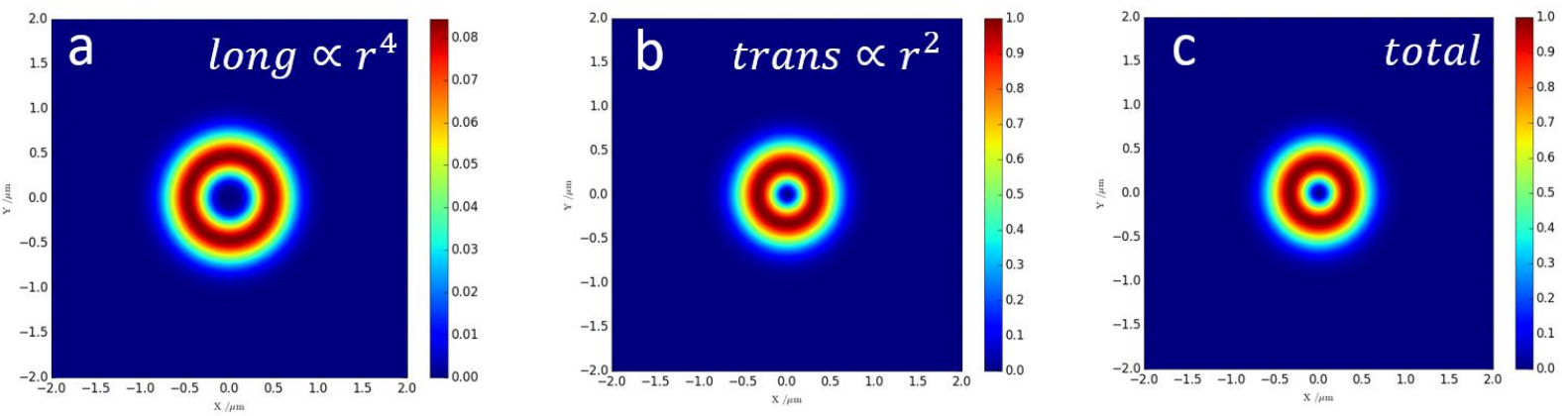

Figure 5: Normalised plots of the individual and total contributions to the rate of single-photon absorption (Eq.(8)) for $\ell= \pm 1, \sigma= \pm 1$. ( $p=0$, $\left.k w_{0} \approx 4\right)$.

\section{CONCLUSION}

LG modes are solutions to the paraxial wave equation, and therefore must strictly be bound to any approximations associated with paraxially propagating light. Detailed conditions of where the paraxial approximation fails for general laser modes can be found in ${ }^{24,25}$. If one wishes to rigorously account for the longitudinal fields of strongly-focused LG beams $k w_{0}<4$, then either (a) the non-paraxial LG solutions should be utilised ${ }^{38,39}$, (b) a systematic expansion in paraxial 
parameter in the spirit of previous studies ${ }^{3,40,41}$ should be performed or (c) explicilt inclusion of the focusing approach with high NA ${ }^{7,20,42,43}$ should be adopted. Importantly, as we have shown, the other terms dependent on the angular momentum properties can be important even for paraxial optical vortices, and their first-order nature means their contributions are completely valid within the paraxial regime.

The field of twisted light and optical OAM has largely been concerned with applications in mechanical nanomanipulation, communications, and quantum information studies - only in the last few years have the unique properties of twisted beams been implemented in atomic and molecular optics and spectroscopy. Here we have highlighted how in such studies, longitudinal fields of optical vortices must be accounted for even for paraxial vortices. Indeed, any application of paraxial vortices clearly require the inclusion of longitudinal fields, particularly for low values of $\ell$ and nanostructures placed close to the so-called vortex singularity. The cases which show most deviation from the purely zeroth-order transverse field description of LG modes appear to be $|\ell|=1, \sigma=0 ; \ell= \pm 1, \sigma=\mp 1, \ell= \pm 2, \sigma=\mp 1$, particularly the first two. Nanostructures with specific orientation with respect to the optical axis have more potential to exhibit larger and more interesting effects with the longitudinal fields than systems of randomly oriented structures. Unlike the strongly-focused case of non-paraxial beams where the longitudinal components can dominate the transverse fields, here it has been shown that for paraxial optical vortices the inclusion of first-order longitudinal fields is still important both qualitatively and quantitatively, as they introduce novel optical interactions with matter as well as alter the electromagnetic fields and corresponding position-dependent intensity structure.

\section{ACKNOWLEDGEMENTS}

KAF thanks the Leverhulme Trust for funding him through a Leverhulme Early Career Fellowship ECF-2019-398.

\section{REFERENCES}

1. Grynberg, G., Aspect, A. \& Fabre, C. Introduction to Quantum Optics: From the Semi-classical Approach to Quantized Light. (Cambridge University Press, 2010).

2. Zangwill, A. Modern electrodynamics. (Cambridge University Press, 2013).

3. Lax, M., Louisell, W. H. \& McKnight, W. B. From Maxwell to paraxial wave optics. Phys. Rev. A 11, 1365 (1975).

4. Adachi, H., Akahoshi, S. \& Miyakawa, K. Orbital motion of spherical microparticles trapped in diffraction patterns of circularly polarized light. Phys. Rev. A 75, 063409 (2007).

5. Zhao, Y., Shapiro, D., McGloin, D., Chiu, D. T. \& Marchesini, S. Direct observation of the transfer of orbital angular momentum to metal particles from a focused circularly polarized Gaussian beam. Opt. Express 17, 2331623322 (2009).

6. Nieminen, T. A., Stilgoe, A. B., Heckenberg, N. R. \& Rubinsztein-Dunlop, H. Angular momentum of a strongly focused Gaussian beam. J. Opt. Pure Appl. Opt. 10, 115005 (2008). 
7. Monteiro, P. B., Neto, P. A. M. \& Nussenzveig, H. M. Angular momentum of focused beams: Beyond the paraxial approximation. Phys. Rev. A 79, 033830 (2009).

8. Bliokh, K. Y. \& Nori, F. Transverse and longitudinal angular momenta of light. Phys. Rep. 592, 1-38 (2015).

9. Babiker, M., Andrews, D. L. \& Lembessis, V. E. Atoms in complex twisted light. J. Opt. 21, 013001 (2018).

10. Klimov, V., Bloch, D., Ducloy, M. \& Leite, J. R. R. Detecting photons in the dark region of Laguerre-Gauss beams. Opt. Express 17, 9718-9723 (2009).

11. Klimov, V. V., Bloch, D., Ducloy, M. \& Leite, J. R. Mapping of focused Laguerre-Gauss beams: The interplay between spin and orbital angular momentum and its dependence on detector characteristics. Phys. Rev. A 85, 053834 (2012).

12. Quinteiro, G. F., Schmidt-Kaler, F. \& Schmiegelow, C. T. Twisted-light-ion interaction: the role of longitudinal fields. Phys. Rev. Lett. 119, 253203 (2017).

13. Andrews, D. L., Jones, G. A., Salam, A. \& Woolley, R. G. Perspective: Quantum Hamiltonians for optical interactions. J. Chem. Phys. 148, 040901 (2018).

14. Andrews, D. L., Bradshaw, D. S., Forbes, K. A. \& Salam, A. Quantum electrodynamics in modern optics and photonics: tutorial. JOSA B 37, 1153-1172 (2020).

15. Babiker, M., Power, E. A. \& Thirunamachandran, T. On a generalization of the Power-Zienau-Woolley transformation in quantum electrodynamics and atomic field equations. Proc. R. Soc. Lond. Math. Phys. Sci. 338, 235-249 (1974).

16. Craig, D. P. \& Thirunamachandran, T. Molecular Quantum Electrodynamics: An Introduction to RadiationMolecule Interactions. (Courier Corporation, 1998).

17. Romero, L. D., Andrews, D. L. \& Babiker, M. A quantum electrodynamics framework for the nonlinear optics of twisted beams. J. Opt. B Quantum Semiclassical Opt. 4, S66-S72 (2002).

18. Forbes, K. A., Green, D. \& Jones, G. A. Relevance of Longitudinal Fields of Paraxial Optical Vortices. ArXiv201209555 Phys. (2020).

19. Bliokh, K. Y., Rodríguez-Fortuño, F. J., Nori, F. \& Zayats, A. V. Spin-orbit interactions of light. Nat. Photonics $\mathbf{9}$, 796-808 (2015). 
20. Iketaki, Y., Watanabe, T., Bokor, N. \& Fujii, M. Investigation of the center intensity of first-and second-order Laguerre-Gaussian beams with linear and circular polarization. Opt. Lett. 32, 2357-2359 (2007).

21. Zhao, Y., Edgar, J. S., Jeffries, G. D., McGloin, D. \& Chiu, D. T. Spin-to-orbital angular momentum conversion in a strongly focused optical beam. Phys. Rev. Lett. 99, 073901 (2007).

22. Andrews, D. L. Quantum formulation for nanoscale optical and material chirality: Symmetry issues, space and time parity, and observables. J. Opt. 20, 033003 (2018).

23. Chen, C. G., Konkola, P. T., Ferrera, J., Heilmann, R. K. \& Schattenburg, M. L. Analyses of vector Gaussian beam propagation and the validity of paraxial and spherical approximations. JOSA A 19, 404-412 (2002).

24. Nemoto, S. Nonparaxial gaussian beams. Appl. Opt. 29, 1940-1946 (1990).

25. Vaveliuk, P., Ruiz, B. \& Lencina, A. Limits of the paraxial approximation in laser beams. Opt. Lett. 32, 927-929 (2007).

26. Quinteiro, G. F., Schmiegelow, C. T. \& Schmidt-Kaler, F. The paraxial approximation fails to describe the interaction of atoms with general vortex light fields. ArXiv Prepr. ArXiv200400040 (2020).

27. Forbes, K. A. On the transfer of optical orbital angular momentum to matter. ArXiv Prepr. ArXiv210110660 (2021).

28. Carnicer, A., Juvells, I., Maluenda, D., Martínez-Herrero, R. \& Mejías, P. M. On the longitudinal component of paraxial fields. Eur. J. Phys. 33, 1235 (2012).

29. Leach, J., Keen, S., Padgett, M. J., Saunter, C. \& Love, G. D. Direct measurement of the skew angle of the Poynting vector in a helically phased beam. Opt. Express 14, 11919-11924 (2006).

30. Allen, L., Lembessis, V. E. \& Babiker, M. Spin-orbit coupling in free-space Laguerre-Gaussian light beams. Phys. Rev. A 53, R2937 (1996).

31. Santamato, E. Photon orbital angular momentum: problems and perspectives. Fortschritte Phys. Prog. Phys. 52, $1141-1153$ (2004).

32. Wang, J., Castellucci, F. \& Franke-Arnold, S. Vectorial light-matter interaction: Exploring spatially structured complex light fields. AVS Quantum Sci. 2, 031702 (2020).

33. Angelsky, O. V. et al. Structured Light: Ideas and Concepts. Front. Phys. 8, (2020).

34. Novotny, L., Beversluis, M. R., Youngworth, K. S. \& Brown, T. G. Longitudinal field modes probed by single molecules. Phys. Rev. Lett. 86, 5251 (2001). 
35. Barron, L. D. Molecular light scattering and optical activity. (Cambridge University Press, 2009).

36. Forbes, K. A. \& Andrews, D. L. Orbital angular momentum of twisted light: chirality and optical activity. J. Phys. Photonics (2021).

37. Andrews, D. L. \& Thirunamachandran, T. On three-dimensional rotational averages. J. Chem. Phys. 67, 5026-5033 (1977).

38. Barnett, S. M. \& Allen, L. Orbital angular momentum and nonparaxial light beams. Opt. Commun. 110, 670-678 (1994).

39. The angular momentum of light. (Cambridge University Press, 2012).

40. Agrawal, G. P. \& Pattanayak, D. N. Gaussian beam propagation beyond the paraxial approximation. JOSA 69, 575578 (1979)

41. Couture, M. \& Belanger, P.-A. From Gaussian beam to complex-source-point spherical wave. Phys. Rev. A 24, 355359 (1981).

42. Bokor, N., Iketaki, Y., Watanabe, T. \& Fujii, M. Investigation of polarization effects for high-numerical-aperture first-order Laguerre-Gaussian beams by 2D scanning with a single fluorescent microbead. Opt. Express 13, 10440 (2005).

43. Bliokh, K. Y. et al. Spin-to-orbital angular momentum conversion in focusing, scattering, and imaging systems. Opt. Express 19, 26132-26149 (2011). 